\title{
O.S.P.
}

L'orientation scolaire et professionnelle

$46 / 2$ | 2017

Adolescence et orientation (2)

Messing, K. Les souffrances invisibles.

Montréal : Ecosociété.

Ginette Francequin

CpenEdition

Journals

Édition électronique

URL : http://journals.openedition.org/osp/5453

DOI : 10.4000/osp.5453

ISSN : 2104-3795

Éditeur

Institut national d'étude du travail et d'orientation professionnelle (INETOP)

Édition imprimée

Date de publication : 12 juin 2017

ISSN : 0249-6739

Référence électronique

Ginette Francequin, " Messing, K. Les souffrances invisibles. ", L'orientation scolaire et professionnelle [En ligne], 46/2 | 2017, mis en ligne le 01 juin 2019, consulté le 25 septembre 2020. URL : http:// journals.openedition.org/osp/5453; DOI : https://doi.org/10.4000/osp.5453

Ce document a été généré automatiquement le 25 septembre 2020.

(c) Tous droits réservés 


\section{Messing, K. Les souffrances invisibles.}

Montréal : Ecosociété.

Ginette Francequin

\section{RÉFÉRENCE}

\section{K. Messing}

Montréal : Ecosociété.

1 Cet ouvrage de 225 pages en 11 chapitres, écrit avec cœur et humour, est tout d'abord une réflexion approfondie sur le travail en usine, dans le nettoyage, auprès des caissières debout, sur le travail des infirmières et des enseignantes. Et, c'est aussi une présentation biographique de l'auteure, Karen, née en 1943, dans un milieu de cadres qui l'autorise très vite à observer le travail en usine.

2 Karen Messing, que j'ai rencontrée il y a une bonne quinzaine d'années à Paris et à Montréal, est reconnue sur plusieurs continents comme généticienne puis est devenue " ergonome ", ses dernières études étant réalisées au Cnam ${ }^{1}$ à l'Institut national d'études sur l'orientation et le travail. Elle est connue notamment en qualité de spécialiste des questions de la santé des femmes au travail, dévoilant avec intelligence fine et émotion « l'invisible qui fait mal ».

3 Elle a 33 ans, lorsqu'elle arrive à Montréal, à l'UQAM, université publique, où le département de biologie existait depuis les années 1970: encouragée par Donna Mergler, professeure de physiologie, elle se « lance » auprès des ouvriers des raffineries de phosphate, soumis aux émissions radioactives. Ces deux femmes engagées vont créer CINBIOSE ${ }^{2}$ qui va séduire nombre de syndicats européens qui rapidement font traduire en six langues les résultats sur « genre et santé au travail » ${ }^{3}$.

4 Karen sait que des rencontres scientifiques ont été importantes : avec Donna Mergler, Ana Maria Seifert, Nicole Vézina, Johanne Leduc, Marie Eve Thibaut, Katherine Lippel, Stéphanie Premji, Jill Hanley et avec toutes les syndicalistes, et en France avec l'ANACT 
(Florence Chappert et nombre d'ergonomes et de sociologues du Cnam qui inspire fortement ses analyses : citons par exemple Laurent Vogel et Patricia Romito, Catherine Cailloux-Teiger, Ghislaine Donio-Shaw ou encore Danièle Kergoat, Annie ThébaudMony).

L'époux de Karen, Pierre Sormany, est important car ce journaliste de l'environnement, solide, critique, engagé, est fort encourageant. Mais en 2008, à la retraite d'« enseignante» de Karen, l'UQAM pousse CINBIOSE «scientifique» vers le département de communication. S'est-elle trop intéressée avec ses étudiant.e.s à ceux et à celles qui bossent au "bas de l'échelle »?

6 Le concept de "fossé empathique" arrive très vite dans l'ouvrage comme un questionnement tenace : les chercheur.e.s en santé au travail manquent-ils d'empathie envers les gens de statut social inférieur? C'est touchant d'imaginer une fillette qui se demande à 10 ans si les ouvrières ne s'ennuient pas à souder des fils rouges, bleus, jaunes au bon endroit sur chacun de ces appareils. La question de l'ennui introduit l'esprit critique qui va l'agiter encore plus à 17 ans, lorsqu'elle se fait embaucher en librairie et en restauration comme serveuse « très mauvaise » dit-elle : retenir les noms de code, négocier avec les commis de cuisine qui la chahutent, c'est une humiliation mentale, car le cerveau de l'intello ne retient rien, il faut autre chose : de l'expérience, du savoir-faire.

7 Ce qui va choquer de suite Karen, dès les premières enquêtes en recherches qu'elle aborde en généticienne humaine, c'est la lâcheté des chercheur.e.s qui se dérobent (comme le professeur surnommé "Ivy»), en quête de titres et avec peur de sanctions, alors qu'il faudrait enquêter, dénoncer les produits dangereux utilisés en usines. En effet, des produits toxiques imprègnent les poumons, les gencives, les habits qu'on rapporte à laver en machines à la maison : les ouvriers apprennent les risques encourus par le journal, car le syndicat a fait appel à Karen qui constate (grâce à un dentiste éclairé par des mâchoires fragiles de patients) que des problèmes de santé se transmettent avec des anomalies, des handicaps aux enfants.

8 La lâcheté de certains fait que la confiance entre travailleurs, travailleuses et recherche universitaire est sujette à perte de confiance, même si Jeanne Stellman avait tiré la sonnette d'alarme à propos de la radioactivité, en écrivant Perdre sa vie à la gagner, tout comme l'avait fait le journaliste du Devoir, Louis-Gilles Francoeur, en mai 1993.

9 C'est avec l'aide de femmes chercheures qu'elle mobilise que Karen se lance alors dans la formulation d'un partenariat UQAM-syndicats (avec la syndicaliste Micheline Boucher) pour faire des recherches, malgré l'absence de littérature scientifique sur le sujet «santé et toxiques" et malgré les pressions pour l'intimider et les contreexpertises "plus ou moins achetées" par des employeurs. De cette période, Karen apprend ce qu'est l'angoisse des ouvriers, la terreur d'une femme contaminée, le dévouement des syndicats : ceci va guider tout son travail et son engagement des années de recherches qui suivent (page 31).

Six chapitres suivront: Le monde invisible du nettoyage; Debout, immobiles; Le cerveau des travailleurs à bas salaire; Le travail d'équipe, une réalité invisible; La violation de domicile; Des enseignants et des chiffres; Devenir une scientifique. Sur les crabes, la douleur et le scepticisme des chercheur.e.s ; Les orteils du statisticien et le fossé empathique dans la littérature scientifique; et enfin, dernier chapitre: Des chercheur.e.s à l'écoute? 
11 Tous les titres sont riches de sens, clairs quant à leur sujet de réflexion.

12 Les ouvriers du nettoyage en milieu hospitalier poussent Karine à devenir ergonome. Elle comprend que non seulement l'exposition aux produits radioactifs est nuisible, mais que, de plus, l'effort de soulever des charges en postures inconfortables, ajouté à la tension et au stress, va nuire aux femmes enceintes et aux fœtus.

13 En ce qui concerne les travailleurs du nettoyage, deux éléments retiennent notre attention :

14 - aucune étude sur le thème, que ce soit à propos des salaires ou des conditions de travail ;

15 - la misogynie du milieu de travail : le ménage est en deux phases distinctes, soit lavage à la serpillière des sols carrelés, poubelles à vider et toilettes à nettoyer vite, en particulier dans les trains pour les femmes, ou sols cirés avec balayeuse-cireuse électrique pour les hommes ;

16 - l'invisibilité de ces femmes réduites à ne pas être vues, mais qui tiennent aussi à rester invisibles, sauf lorsqu'elles portent une belle veste en cuir ou un corsage doré ;

17 - comment Karen découvre l'ergonomie, le plaisir d'observer le travail, de comprendre (questionnaire en présence, entretien, observation des postures) ;

18 - l'action qui mène au «retrait préventif» pour femmes enceintes et pour cause d'allaitement ;

19 - l'alliance chercheures et syndicalistes, délicate à se mettre en place, mais qui se fait d'abord, entre femmes ;

20 - les pressions et menaces pour ne pas dévoiler les problèmes dans des rapports, colloques ou articles ;

21 - se poser des questions éthiques, avoir des critères pour rendre visible le travail invisible des travailleuses du « bas de l'échelle ».

22 Le chapitre 3 traite de Debout, immobiles et commence par Renée, le travail de caisse en banque et le mal de dos. La question du respect : quand la chercheure peut s'asseoir, la caissière de banque reste debout. Pourtant, des vendeuses sont assises en Suède, en Grèce, en France, en Chine, au Cameroun, au Brésil et au Pérou ! Et malgré le règlement (article 1.7.1 du règlement québécois). Et si l'une a gagné, 24 ans plus tard, Karen observe que les choses n'ont pas changé le retard.

23 La Commission des normes et les autres organismes ne font pas leur boulot : détecter en quoi travailler debout peut entraîner une myriade de troubles divers. La " pire » caisse, la plus étroite, celle qui autorise le moins de mouvements, est bien décrite par les travailleurs, mais jamais par la science, et il faut noter que le travail debout est ordinaire en Amérique du Nord, alors qu'ailleurs (France, Suède), on travaille généralement en posture assise.

24 Puis, Karen observe huit vendeuses durant une journée de travail. Sur ces huit vendeuses, cinq ont mal aux pieds, posent le pied sur l'autre, lèvent un pied, se frottent le dos; elles souffrent en silence et ignorent la règle de pause assise de dix minutes. Mais lorsque Karen expose ses observations à des universitaires / clientes, celles-ci font remarquer qu'elles ne supporteraient pas de voir leur caissière assise ; ce qui permet à Karen Messing de développer son concept de « fossé empathique ». 

l'absence de supervision (cas du nettoyage), mais lorsqu'on fait décliner les effectifs de maladie nosocomiale surgit l'idée de faire moins de coupures. Surtout Karen s'interroge sur le fait que personne ne se révolte, d'autant que la pause est accordée aux fumeurs, mais pas aux femmes fatiguées qui aimeraient juste s'asseoir. Ce qui semble primordial reste le salaire, et les personnes de «rang subalterne» se taisent pour pouvoir travailler plus, qu'on leur offre des heures supplémentaires à faire, sans déplaire à la clientèle.

Le chapitre 4 traite du cerveau des travailleurs à bas salaire. Elle fut séduite par l'ergonomie fondée par des chercheurs parisiens du Cnam (François Guérin, Antoine Laville, François Daniellou, Jacques Durrafourg et Alain Kerguelen) et a développé l'observation du langage et des postures qui sous-tendent l'activité (pour les opératrices de machines à coudre : hauteur de l'étagère, réglage de la machine, qualité d'écoute du contremaître). Catherine Teiger reste son ergonome modèle. Karen s'est débrouillée pour avoir les fonds qui permettent de faire venir des chercheures et syndicalistes de Finlande, du Nicaragua, de France, de Thaïlande, des États-Unis, d'Afrique du Sud sur le thème du travail répétitif des femmes lorsqu'elles cousent des gants de protection pour l'industrie. Catherine Teiger avait rencontré des « couseuses mal en point ", qui intégraient l'usine à 17 ans et n'avaient pas plus de 25 ans ; payées aux pièces, qui produisaient un gant bien ajusté toutes les 40 secondes : 900 gants par jour produits en position inconfortable. Catherine Teiger avec Nicole Vézina et Karen Messing vont développer les " compétences méconnues »; et ce groupe avec Ghislaine Doniol-Shaw et Colette Bernier suivent le travail à la chaîne, que ce soit en couture, en emballage de gâteaux, et si apparemment trois jours suffisent pour apprendre le métier, puis deux mois pour acquérir la vitesse, il faut en fait deux ans pour réparer la machine en panne, aider les collègues qui débutent, anticiper les problèmes. Tout changement dans l'organisation du travail risque de perturber gravement la santé des travailleurs, surtout si la modification «nuit au salaire d'un autre travailleur». Le travail sur chaîne de montage, comme le travail de serveuse, pose des défis cognitifs et émotionnels si des clients se plaignent.

Karen cite une étude de 2005, suggérée par le syndicat de la restauration pour montrer « leur intelligence » dans un métier exigeant au physique comme au mental, avec des TMS, des maux de pieds, de bras, d'épaules. Des stratégies de défense se mettent en place, pour tenir physiquement face aux pas à faire, au transport de charges, pour mémoriser les commandes, se donner des défis émotionnels face au client déprimé, au client excité, voire un peu graveleux, d'autant que le pourboire est un élément significatif du salaire en Amérique du Nord, déjouer les caprices de rapidité ou la lenteur des collègues en cuisine. Parfois, supporter le bruit et les lumières excitantes s'ajoute au travail comme le décolleté de l'uniforme qui découvre plus ou moins les seins, jouant sur le pourboire qui contribue à accentuer l'écart social entre serveuses et clientèle. Karen insiste à juste titre sur cette question du pourboire et de son lien à la santé, car il disqualifie le salaire, le travail, la qualification et valorise la hiérarchie, et Karen aimerait apporter des solutions réelles et dignes aux difficultés des serveuses.

Dans le cinquième chapitre, c'est le travail d'équipe qui va être analysé, car deux chercheures en binôme sont des alliées du collectif : Ana-Maria la sensible, chaleureuse, capable d'écouter et d'aider physiquement les personnes de qui elle analyse le travail et Karen, la convaincue de l'ergonomie, qui lie des partenariats durables avec les 
syndicats : CSN (le milieu hospitalier) et FTQ (les caissières, les banques) depuis 1992 puis la CEQ (enseignant.e.s du primaire). D'où, L'Invisible qui fait mal. C'est aussi comment l'union fait la force, au niveau financier, puisque des succès notoires sont arrivés : fiche et brochure pour l'ONU, chaire de recherche de Katherine Lippel, études plus faciles car financées. Ces études permettent de mettre l'accent sur les peurs et le stress : le vol, la violence, la confiance, sur la gêne éprouvée face aux problèmes sociaux, aux chèques d'aide sociale. Elles mettent en valeur la gêne de la course au gain des étoiles, car c'est celle de l'évaluation et de la méritocratie, contre le travail en commun et l'entraide face aux horaires atypiques et aux réorganisations qui malmènent les équipes.

Le travail des infirmières est parfaitement décrit et a beaucoup plu à une infirmière parisienne qui a lu le livre, reconnaissant les mêmes soucis ici et au Québec, car le manque de temps pour la relation humaine est évident, et chaque remaniement du personnel hospitalier est une baisse de qualité de soins.

Le travail dans les abattoirs, dans l'école, dans les usines est bien décrit suite à de multiples études parfaitement cadrées et approfondies de Johanne Prévost, Alain Lajoie, Nicole Vézina, Céline Chatigny.

31 Dans le sixième chapitre, le titre Violation de domicile intrigue un peu, et on sent qu'il s'agit des "domestiques ", vivant un peu comme les servantes de la fin du XIX ${ }^{\mathrm{e}}$ siècle. La privation de sommeil des dames de ménage qui travaillent la nuit est analysée par Karen à partir de sa propre expérience de femme et de mère. Être parent ou pas est un facteur qui joue sur l'appréciation des horaires et conditions de travail, et la vie familiale s'immisce, surtout dans les métiers pénibles ou à horaires rigides.

Quels sont les rapports des salarié.e.s avec la hiérarchie? Les questionnaires, les observations sur le terrain, les entretiens sont révélateurs des paradoxes concernant des jours de congé mal attribués, le manque de temps pour vivre avec sa famille.

Le chapitre sur les enseignantes est argumenté de chiffres, car cette question intéresse que ce soit sur les conditions de travail, la formation des professeurs, la réforme du programme, les journées pédagogiques, la religion à l'école et les réussites attribuables aux enseignant.e.s. Le travail hors classe est souvent non compris par les parents, aucune compassion pour leur travail vu comme un rôle de gardiennage, aucun intérêt pour leur bas salaire, les enseignants sont mal vus, reçoivent de la violence, de l'intimidation des parents, de l'administration et n'ont pas de soutien du gouvernement : stress, difficultés devant les élèves qui ne parlent pas la langue scolaire.

Le chapitre 8 retrace le parcours qui permet de devenir une scientifique : une étudiante de Karen, Micheline Cyr, fille d'un laveur de vitres et d'une femme de ménage malade, travaille d'abord, comme son mari, pour pouvoir reprendre ses études et faire sa maîtrise en biologie. Sa famille d'origine s'étonne qu'elle étudie encore à 25 ans. Elle renoncera finalement au doctorat pour travailler comme conseillère en centre d'hébergement de nuit pour femmes itinérantes: elle développe ce service sur le Québec. L'histoire personnelle de Mimi lui donne une écoute très fine et interroge Karen, elle qui est fille d'un "cadre supérieur » et d'une artiste de gauche et interroge l'auteure sur les moyens de réaliser des études universitaires et comment... L'une, Karen, fait Harvard, sciences naturelles, travaille en 1962 avec le professeur Nash et lit Betty Friedan, La Femme mystifiée, et décide de devenir chimiste " comme lui ». Elle veut faire médecine, mais les femmes avec enfants sont refusées à l'inscription. Grâce à la rencontre avec Danielle Saint Aubin, une amie étudiante en chimie, elle apprend le 
français, elle fait une maîtrise avec un professeur de génétique, se fait malmener parce que mère de famille par une travailleuse sociale. Elle part en voyage d'études à Cuba avec son professeur et rencontre Len Radinsky, spécialiste de l'anthropologie physique, qui la fascine, car elle comprend avec lui que l'intellectuel peut aider à transformer la société, aider le tiers-monde! Travaillant avec Lynn et Harry, elle découvre les inégalités de traitement entre les chercheur.e.s, hommes-femmes. Et les difficultés des mères de famille pour concilier les études, les recherches et la maternité! ses angoisses, celles de Carol, monoparentale avec trois enfants qui assure son métier et ses études : femmes et sprint en permanence. Un bus est libre à la crèche, mais on ne s'en servira pas, car "les mères doivent apprendre à s'organiser». Elles sont « irresponsables » de faire une telle demande, alors qu'elles font des pieds et des mains pour arriver à tout faire ! 1975 : finalement, Karen est enfin docteure, après cinq années de travail. En 1976, en post-doc, elle a un poste à Montréal. Heureuse, car l'UQAM se libère des contraintes et met en place des expérimentations.

L'IRSST (Institut de recherche en santé au travail) est mis en place par le gouvernement du Québec : 1982, elles se joignent à deux collègues hommes, Michel et Jacques. Mais ils vont s'opposer à une idée de recherches sur femmes et travail. Finalement, Donna et Karen vont codiriger « la prévention préventive ", comme on se moque d'elles.

Dans le chapitre 9, en 2006, colloque à Saint-Jean, Terre-Neuve, Karen pleure... car, sur les crabes, la douleur et le scepticisme des chercheur.e.s. Asthme des femmes, et TMS, et amiante... et le fossé empathique qui est le refus des chercheur.e.s de parler d'une situation à laquelle ils.elles ne croient pas. Et Karen repart en guerre contre les méthodes inadéquates et lance EQCOTESST, avec 5000 entretiens et 11 chercheur.e.s au dépouillement. Le rapport fut enfin publié le 20 septembre 2011, malgré la pression de trois employeurs sur la diffusion des résultats; mais elle aura du mal à obtenir une autre recherche. Rigueur et objectivité... sont, elles, une question de points de vue. "Mais on nous apprend à ne pas écouter les travailleurs ", dit-elle. Et les résistances sont énormes pour laisser travailler chercheur.e.s et syndicats ensemble.

Dernier chapitre : les orteils d'un statisticien et le fossé empathique dans la littérature scientifique : le risque acceptable. Pour qui ? Qui décide? Qui paie? Les chercheur.e.s ont besoin de partenaires. Karen part d'un exemple pris dans sa vie personnelle, où avec son conjoint Pierre, en avril 2012, ils sont en vélo au Pérou, et un chien mord Pierre, et pour conjurer le risque de la rage, le couple mesure le risque acceptable (seuil à 0,05 ) d'une intervention à l'étranger et de lier tout ceci de manière amusante aux orteils de Fischer qui déterminent quels médicaments sont jugés efficaces. L'idée de Fisher est qu'on peut établir une différence significative entre deux groupes si la probabilité qu'ils soient identiques est inférieure à 1 sur 20 (un seuil alpha de 0,05 et un seuil bêta de 0,20 ). Ceci est utilisé pour déterminer ce qui est dangereux ou pas pour les travailleurs (lien entre mal de dos et charges lourdes). Cependant, Karen est critique par rapport à Fisher, car il a eu l'idée en ne s'occupant que d'un pied, celui qu'il regardait, et pas des deux! La question de la fatigue et des risques d'accidents aurait été mieux posée s'il avait regardé ses deux pieds!

Et si les travailleurs et leurs familles fixaient eux-mêmes le seuil de tolérance des charges? se demande Karen. Elle en revient aux compétences de Katherine Lippel, de Nicole Vézina qui observe trois employées des postes avec douleurs chroniques. Elle dénonce aussi l'utilisation malhonnête du vocabulaire des chercheur.e.s par des gens de cour lorsque le.la chercheur.e prend des précautions au conditionnel ou nuance 
ses verbes (exemple : laissent entrevoir... peuvent aider à). On leur a appris à ne pas faire de déclarations trop catégoriques, trop "militantes", mais à garder un ton réservé.

C'est pourquoi Karen Stéphanie et Katherine décident de faire une étude de 20 articles sur le lien entre TMS et conditions de travail dans deux revues dont l'une est plus proche des employeurs que l'autre. Elles constatent de fait un double langage qui fausse la réflexion sur la santé des travailleurs, à leur désavantage. Par ailleurs, elles se rendent compte que l'idée "qu'on ne les croit pas, que les femmes inventent des douleurs, des causes de travail» existent et donc faire la preuve scientifique est complexe, en minant l'empathie envers les travailleurs.

Enfin, le chapitre 11, intitulé Des chercheurs à l'écoute?, aborde le concept de «fossé empathique » et la difficulté de faire évoluer les conditions de travail. Elle se réjouit de l'existence du retrait préventif pour grossesse ou allaitement, grâce à l'obstination des travailleuses. Lorsque la prévention bouge pour quelques personnes, elle finit par bouger pour tout le monde, et c'est un acquis, comme le droit de s'asseoir, mais il y a souvent des contre-expertises, des stratégies pour gagner plus d'argent, même si c'est aux dépens de la santé des travailleurs. Heureusement, des travailleurs restent courageux et tenaces, comme Robert qui se meurt d'un cancer de l'estomac, mais va jusqu'au bout, écrivant une lettre sur la santé-sécurité au travail.

Karen pense qu'on entend ce qu'on veut bien entendre et que les politiques publiques en recherche doivent être bien orientées (elle pense à l'amiante, à la fatigue qui rend triste) sans crainte de perdre ses subventions, mais en ayant le courage de se battre pour les garder.

Ce livre souligne bien les enjeux, les difficultés et l'importance du travail interdisciplinaire, fait l'éloge de l'ergonomie lorsqu'elle ose accomplir son travail d'observation, avec des rapports bien articulés. Ceci veut dire oser résister aux attaques, aux pressions, aux insultes, se battre malgré tout. Les chercheur.e.s ont besoin de partenariats universités-syndicats pour innover, avoir plus de financements, de temps, de cadres de fonctionnement pour établir des relations durables et critiques avec les communautés, et que les syndicats se renseignent, se documentent pour éviter de ne pas être sensibles aux arguments patronaux qui les bluffent souvent pour discréditer les chercheur.e.s (e.g. "vos chercheur.e.s publient dans les mauvaises revues ".

Karen, exigeante, demande aussi le soutien de la population aux recherches, que les " gens » se réveillent pour mieux prévenir sur la santé au travail, le respect du savoir et des efforts accomplis.

44 Un livre à faire connaître, à lire !

\section{NOTES}

1. . INETOP, 41 Rue Gay-Lussac à Paris. 
2. CINBIOSE : Centre de recherche interdisciplinaire sur la biologie, la santé, la société et l'environnement. Ergonomes, sociologues, biologistes, juristes et représentants syndicaux.

3. . Comprendre le travail pour le transformer. Institut syndical européen.1999. 